\title{
Oxygen-dependent alginate synthesis and enzymes in Pseudomonas aeruginosa
}

\author{
JORGE H. LEITÃo and I. SÁ-CORREIA* \\ Laboratório de Engenharia Bioquímica, Instituto Superior Técnico, Av. Rovisco Pais 1096 Lisboa Codex, Portugal
}

(Received 26 June 1992; revised 2 November 1992; accepted 5 November 1992)

\begin{abstract}
Alginate production by the highly alginate-producing $P$ seudomonas aeruginosa $8821 \mathrm{M}$ was maximal at a dissolved oxygen tension (DOT) of $5 \%$ of air saturation. Lower DOT limited growth and alginate synthesis. At higher DOT values up to $70 \%$ of air saturation, the specific alginate production rate decreased. Nevertheless, the molecular mass of the alginate increased at higher aerations, as indicated by the viscosity of solutions of the isolated biopolymer. The specific activity of the four enzymes leading to GDP-mannuronic acid formation, phosphomannose isomerase (PMI), phosphomannomutase (PMM), GDP-mannose pyrophosphorylase (GMP) and GDP-mannose dehydrogenase (GMD), increased with DOT of up to $25 \%$. At higher DOT, however, only GMP and GMD maintained their maximum values. Changes observed at high oxygen concentrations in the relative activities of PMI and GMP, which are activities of the same bifunctional protein, were attributed to the much higher sensitivity of PMI activity to irreversible oxidative inactivation. The less pronounced decrease of PMM activity at high DOT correlated with an intermediate sensitivity to oxidative inactivation, but could also be related to sequential induction of PMM by the product of the PMI reaction. Thus, oxygen-dependence of alginate synthesis was at least partially the effect of DOT on GDP-mannuronic acid formation. Optimal aerations for maximal alginate production (DOT $=5-10 \%$ ) were below the aeration level $(70 \%)$ that led to the highest viscosity. These results suggest that, like GMD, polymerization activity is not very sensitive to oxidative inactivation and they are consistent with the hypothesis that polymerization is dependent on GMD activity, or is regulated in a similar way.
\end{abstract}

\section{Introduction}

Although culture aeration is important for the production of exopolysaccharides of commercial significance, not all microbial cells require maximal aeration for optimal production (Sutherland, 1990). Optimization of operational variables such as mixing and aeration is particularly important in exopolysaccharide fermentation since high product yield leads to high viscosity of the fermentation liquor, causing a marked decrease in aeration efficiency due to a much lower mass transfer rate between the air bubbles and the broth. Beside its role as the terminal electron acceptor in aerobic respiration, oxygen is required in both the synthesis of uronic acid residues of the biopolymers and, indirectly, the reoxidation of the reduced pyridine nucleotides generated.

\footnotetext{
*Author for correspondence. Tel. 18473412 ext. 1233; fax 18480072.

Abbreviations: DOT, dissolved oxygen tension; PMI, phosphomannose isomerase; PMM, phosphomannomutase; GMP, GDPmannose pyrophosphorylase; GMD, GDP-mannose dehydrogenase.
}

However, no information is available on the effect of dissolved oxygen tension either on the expression of genes encoding enzymes indispensable to exopolysaccharide synthesis, on enzyme stability, or on the yield and characteristics of the exopolysaccharides produced.

Mucoid $P$. aeruginosa strains, which produce copious amounts of alginate, are being intensively studied because of their relevance to respiratory infection in patients with cystic fibrosis (DeVault et al., 1989). Environmental factors such as nitrogen starvation and nitrogen source, general nutrient deprivation, medium osmolarity and carbon source have been reported to affect the transcriptional activation of the alginate gene algD, encoding GDP-mannose dehydrogenase (GMD) (Deretic et al., 1990, 1991; DeVault et al., 1989, 1991). This enzyme catalyses the irreversible oxidation of GDPmannose to GDP-mannuronic acid, which is the activated precursor for alginate polymerization. Transcriptional activation of the $\operatorname{alg} D$ gene is involved in the expression of the mucoid phenotype (Deretic et al., 1987). Recently we demonstrated that spontaneous variation in the level of alginate synthesis by highly alginate-producing $P$. aeruginosa strains is associated 
with changes in the activity of the other three enzymes leading to GDP-mannuronic acid synthesis: phosphomannose isomerase (PMI), which catalyses the reversible isomerization of fructose 6-phosphate (F-6-P) to mannose 6-phosphate (M-6-P), phosphomannomutase (PMM) which catalyses the conversion of M-6-P to mannose 1-phosphate (M-1-P) and GDP-mannose pyrophosphorylase (GMP) which catalyses the conversion of M-1-P to GDP-mannose (Leitáo et al., 1992). Temperature was found to influence strongly the yield and properties of the alginate produced by $P$. aeruginosa 8821M (Alves et al., 1991; Leitão et al., 1992). This was associated with regulation of the four alginate enzymes PMI, PMM, GMP and GMD, although the involvement of enzymes that catalyse steps downstream from the formation of GDP-mannuronic acid had also to be considered to explain in full the effects of temperature on alginate synthesis (Leitão et al., 1992).

Substantial differences in the yield of alginate synthesis by mucoid and non-mucoid Pseudomonas aeruginosa strains at two different oxygen tensions, reflective of the right versus left cardiac circuits in vivo ( 40 vs $80 \mathrm{~mm} \mathrm{Hg}$, respectively), were reported by Bayer et al. (1990). They suggested that this was associated with the worse prognosis for cases in patients experiencing left-side bacterial endocarditis as compared with those with rightside infection (Bayer et al., 1990). The results also suggested that variations in oxygen tensions could be one of the trigger mechanisms for the up-regulation of alginate synthesis in non-mucoid $P$. aeruginosa strains (Bayer et al., 1990).

In the present work, the effect of DOT on alginate enzymes and alginate synthesis by the highly alginateproducing $P$. aeruginosa $8821 \mathrm{M}$ (Alves et al., 1991) was investigated.

\section{Methods}

Bacterial strain and media. The highly alginate-producing variant Pseudomonas aeruginosa 8821M (Alves et al., 1991) was used. Phenotypically well-defined colonies of $8821 \mathrm{M}$ grown on PIA (Pseudomonas Isolation Agar, Difco) plates were used to inoculate LB liquid medium [ $10 \mathrm{~g}$ Bacto-peptone (Difco), $5 \mathrm{~g}$ yeast extract (Difco) and $5 \mathrm{~g}$ $\mathrm{NaCl}$ (Merck) in 1 litre]. Cultures were grown overnight with agitation (200 r.p.m.) at $30^{\circ} \mathrm{C}$ to prepare the inocula. Volumes of inocula, calculated in order to obtain an initial $\mathrm{OD}_{640}$ of $0.30 \pm 0.02$, were centrifuged and cells were resuspended in $5 \mathrm{ml}$ fermentation medium and used to inoculate the fermenter. Fermentation medium consisted of $\mathrm{LB}$ with the addition of $10 \mathrm{~g}$ glucose per litre and $\mathrm{MgCl}_{2}$ and $\mathrm{MnCl}_{2}$ to $0.5 \mathrm{~mm}$ each (Martins et al., 1990).

Culture conditions. Batch fermentations were carried out in a BIOLAB fermenter (B. Braun, Germany) The working volume of the vessel was 1.851 and temperature was maintained at $30.0 \pm 0.1{ }^{\circ} \mathrm{C}$. Dissolved oxygen tension (DOT) $(0,1,5,10,25,40$ and $70 \%$ relative to fermentation medium air saturation at $30^{\circ} \mathrm{C}$ ) was measured using an oxygen electrode and controlled automatically by the sterile air input flow rate. When the air input was not sufficient to maintain the DOT at the set point, agitator speed was increased from 250 r.p.m. (the agitation used in normal operating conditions) to 600 r.p.m. This happened when culture ${ } D_{640}$ reached about $0 \cdot 8$, except at the two lowest oxygen concentrations. For the lowest DOT used, the air flow rate was kept constant $\left(100 \mathrm{ml} \mathrm{min}^{-1}\right.$, equivalent to $0.054 \mathrm{vol}$. air per vol fermentation broth $\min ^{-1}$, vvm) and the DOT displayed by the oxygen meter was zero or below the zero set-point.

Growth, alginate production and properties. Growth was monitored by measuring the $\mathrm{OD}_{640}$ of the culture. Specific growth rates were calculated by least-square fitting to the linear part of the semi-log growth plots. Alginate concentration was determined after ethanol precipitation from cell-free culture supernatants by the modified carbazole method (Knutson \& Jeanes, 1968) with sodium alginate from Laminaria hyperborea (BDH-Merck) as standard. Results are means of three independent analyses. The viscosity of the aqueous solutions $(3 \mathrm{~g}$ alginate $~^{-1}$ ) and the acetyl content (expressed as acetate content) of the alginates produced were determined as described by Leitão et al. (1992). Results are the means of three independent determinations.

Alginate enzymes. Alginate enzymes were assayed in crude cell extracts prepared from cells harvested at $\mathrm{OD}_{640}=1.52 \pm 0.02$. Assay methods and specific activity units were as described by Leitão $e t$ al. (1992). In order to evaluate the sensitivity of alginate enzymes to oxidative inactivation, crude extracts were incubated at $25^{\circ} \mathrm{C}$ for 0,2 , 5 and 10 min with $0 \cdot 1 \mathrm{M}-\mathrm{H}_{2} \mathrm{O}_{2}$ and the residual activity was immediately determined. In the assay mixtures, a low concentration of $\mathrm{H}_{2} \mathrm{O}_{2}$ $(0.005 \mathrm{M})$ was still present as the result of the crude extract dilutions [50 $\mu \mathrm{l}$ in the final assay volume of $1 \mathrm{ml}$ (Leitão et al., 1992)].

\section{Results and Discussion}

\section{Effects of DOT on alginate production and characteristics}

DOT below $5 \%$ of air saturation limited growth (Figs 1 and 2). At the lowest concentration of oxygen used (considered to be $0 \%$ of air saturation) the aeration rate was kept constant at $0.054 \mathrm{vvm}$. As the biomass concentration increased, the very low initial oxygen concentration of the medium was further reduced. Therefore the growth rate decreased continuously during incubation (Fig. 1a). Alginate concentration at $\mathrm{OD}_{640}$ $1.52 \pm 0.02$ correlated with increasing DOT up to $5 \%$ of air saturation (Figs 1 and 2). At DOT $>5 \%$, alginate synthesis was somewhat inhibited when oxygen concentration increased (Figs 1 and 2). The molecular mass of the biopolymer being synthesized increased at higher dissolved oxygen concentrations, as shown by the higher viscosity of solutions $\left(3 \mathrm{~g} \mathrm{l}^{-1}\right)$ of preparations of the alginates (Fig. 2). The degree of acetylation of alginates synthesized in cultures having DOT values $\geqslant 1 \%$ of air saturation did not vary significantly. The slightly increased acetyl content of alginates produced by cells with slightly reduced growth rates under oxygen limitation was consistent with the growth-rate dependence of acetylation observed by Leitão et al. (1992), who found that at sub-optimal growth temperatures, alginate acetylation decreased linearly with increasing specific growth rate (Leitão et al., 1992). However, at the lowest 


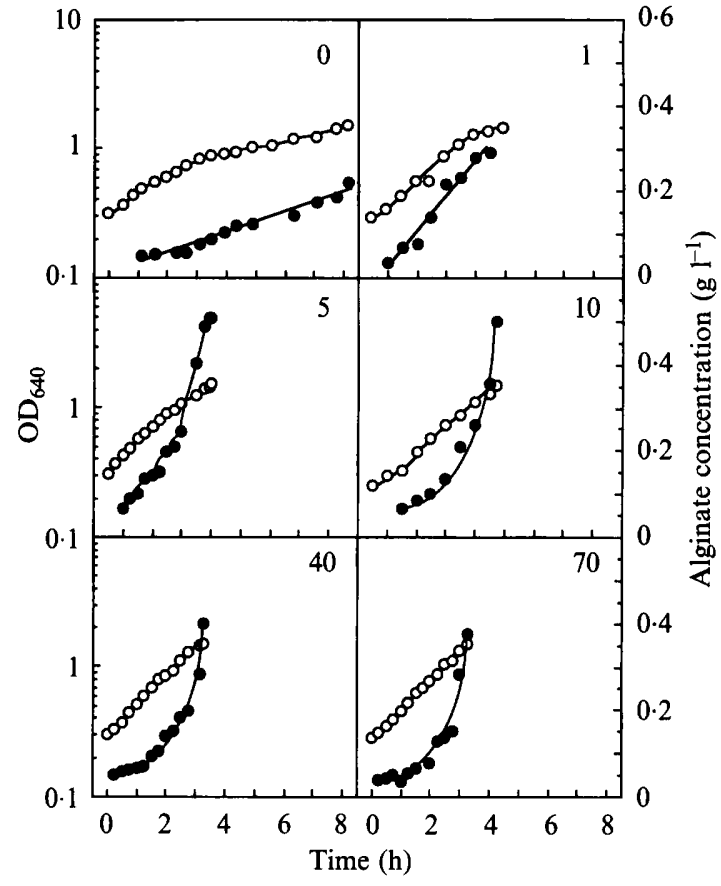

Fig. 1. Production of alginate $(O)$ and culture $\mathrm{OD}_{640}(O)$ for $P$. aeruginosa $8821 \mathrm{M}$ during batch growth at different DOTs: $0,1,5,10$, 40 or $70 \%$ of air saturation (figure inside box).

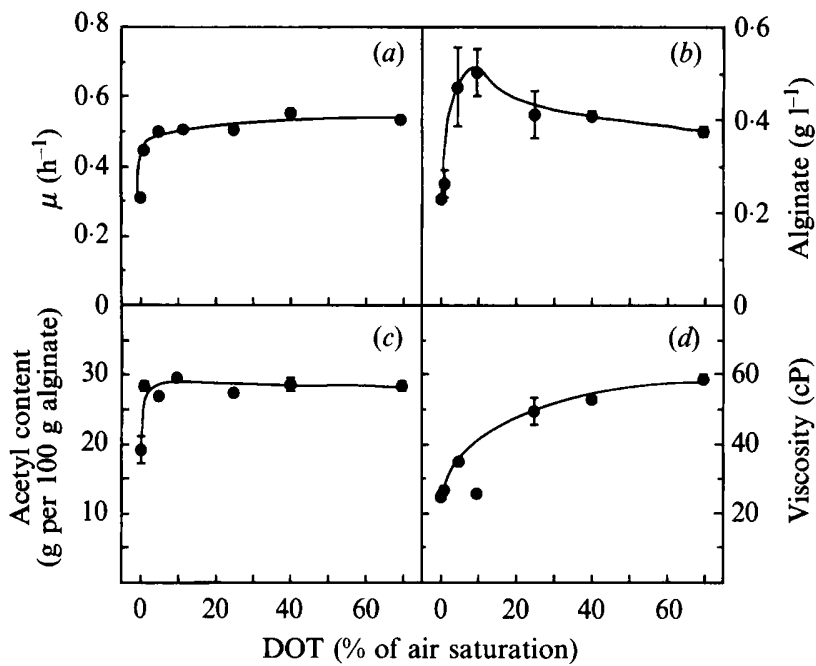

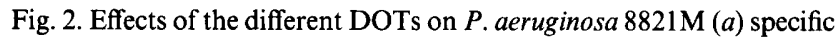
growth rate $(\mu) ;(b)$ alginate concentration at $\mathrm{OD}_{640}=1.52 \pm 0.02 ;(c)$ acetyl content of the alginates; $(d)$ viscosity of $3 \mathrm{~g} \mathrm{l}^{-1}$ alginate solutions (in centipoise, $\mathrm{cP}$; shear rate: $24 \mathrm{~s}^{-1}$ ).

concentration of oxygen used, which severely restricted growth, acetylation was minimal (Fig. 2).

\section{Effects of DOT on the activity of alginate enzymes}

As was observed for alginate production, the specific activities of the four enzymes involved in GDPmannuronic acid formation increased dramatically when

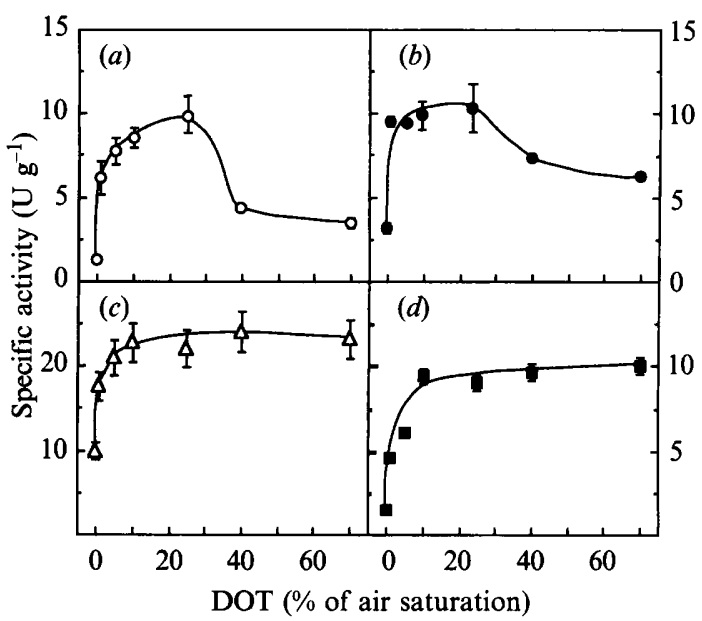

Fig. 3. Specific activities of GDP-mannuronic-acid-forming enzymes of $P$. aeruginosa $8821 \mathrm{M}$ grown at different DOTs. (a) Phosphomannose isomerase (O); (b) phosphomannomutase (O); (c) GDP-mannose pyrophosphorylase $(\triangle) ;(d)$ GDP-mannose dehydrogenase $(\mathbb{\square})$.

the DOT was increased over the range $0-25 \%$ of air saturation (Fig. 3). However, at higher oxygen concentrations, only GMP and GMD maintained the maximum value or smoothly increased (Fig. 3). The decrease of PMI activity at DOT values above $25 \%$ was rather unexpected considering the oxygen-dependence observed for GMP activity (Fig. 3). There is strong evidence that the product of the $\operatorname{alg} A$ gene is bifunctional (Sá-Correia et al., 1987; Shinabarger et al., 1991). This protein appears to catalyse two non-contiguous reactions on the alginate pathway; the isomerization of F-6-P to M-6-P (PMI activity) and the conversion of M-1-P to GDPmannose (GMP activity). Efforts to determine whether these are independent domains of the polypeptide were not conclusive (Shinabarger et al., 1991). Nevertheless, the reactions catalysed by PMI and GMP are very different mechanistically and do not appear to share common substrate binding sites. This was shown by the lack of GMP inhibition by the substrates or products of PMI and vice versa (Shinabarger et al., 1991). Moreover, PMI, but not GMP, was dithiothreitol-sensitive, suggesting that disulphide bonds are necessary for activity (Shinabarger et al., 1991). If these enzymes are activities of the same bifunctional protein, the oxygen-dependent regulation of PMI and GMP activities would be expected to be identical. The most likely explanation for the differences observed in the relative specific activities of PMI and GMP in bacteria grown at different DOTs within the range $0-70 \%$ of air saturation is that the two enzyme domains exhibit different resistances to oxidative inactivation. In order to test this hypothesis, crude cell extracts were incubated with $0 \cdot 1 \mathrm{M}$-hydrogen peroxide and the residual activity of the four alginate enzymes determined. PMI activity was much more sensitive to 


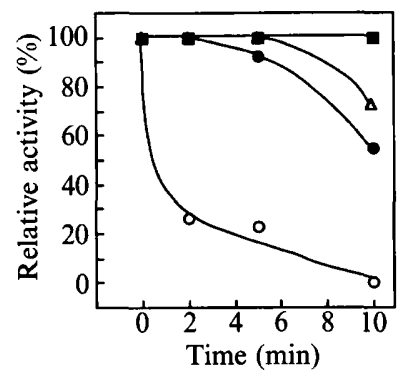

Fig. 4. Residual enzyme activities in crude cell extracts incubated for $0,2,5$ and $10 \mathrm{~min}$ at $25^{\circ} \mathrm{C}$ with $0 \cdot 1 \mathrm{M}-\mathrm{H}_{2} \mathrm{O}_{2}$. Symbols as Fig. 3 .

irreversible oxidative inactivation than the other three alginate enzyme activities under these conditions, having a half-life of less than $1 \mathrm{~min}$, whereas the others retained over $50 \%$ of their activity after 10 min treatment and GMD was completely resistant (Fig. 4).

Oxidative inactivation of some enzymes is triggered by chemical damage of amino acid residues, such as deamination of Asn residues which is followed by hydrolysis of the peptide bond at the Asp so formed, destruction of Met residues and cleavage at the $\beta$ position of Cys residues (Nosoh \& Sekiguchi, 1991). Mutant proteins with non-oxidizable amino acids (Ser, Ala or Leu) replacing the Met residue 222 in Bacillus amyloliquefaciens subtilisin, were resistant to irreversible inactivation by $\mathrm{H}_{2} \mathrm{O}_{2}$ whereas proteins with oxidizable residues (Met or Cys) were readily inactivated (Estell et al., 1985).

Although phosphomannomutase (PMM) appeared to be much more resistant to $\mathrm{H}_{2} \mathrm{O}_{2}$ inactivation than PMI, the activities of the two enzymes responded in similar ways to changes in the DOT of the culture, though PMM activity was slightly less sensitive (Fig. 3 ). The less pronounced decrease of PMM activity at high DOT correlated with an intermediate sensitivity to oxidative inactivation but is also consistent with the model proposed by Sá-Correia et al. (1987) for the sequential induction of PMM by the product of the PMI reaction. Evidence supporting this hypothesis came from the increase in PMM activity in $P$. aeruginosa in response to overexpression of either the $P$. aeruginosa alg $A$ gene or the E. coli pmi gene encoding proteins with PMI-GMP or only PMI activities, respectively (Sá-Correia et al., 1987).

\section{Manipulation of the alginate pathway by varying the DOT}

Reinforcing results from previous work (Leitão et al., 1992), variations in alginate synthesis in $P$. aeruginosa with DOT were associated with changes in the activity of all four enzymes leading to GDP-mannuronic acid formation and not only, or particularly, of GMD. However, studies concerning the environmental control of alginate synthesis have only been based on the regulation of the $\operatorname{alg} D$ promoter (DeVault et al., 1989, 1991; Deretic et al., 1990). The decrease of the alginate yield for DOT $>5 \%$ (Fig. 2) might be partly the result of decreased formation of GDP-mannuronic acid. In fact, both PMI and PMM specific activities decreased at DOT $>25 \%$, although the level of GMD slightly increased and GMP maintained its highest value at high aerations.

Aeration for maximal alginate production $(5-10 \%$ of saturation) was below that leading to alginates with the highest molecular mass $(70 \%)$ as indicated by viscosity measurements (Fig. 2). These results suggest that polymerization activity is not highly sensitive to oxidative inactivation. Since GMD specific activity was highest in cells grown with DOT of $70 \%$, the results are also consistent with the hypothesis that polymerization activity is under a control mechanism similar to that of GMD and/or dependent on its activity (Leitão et al., 1992).

This work was partially supported by Grant BIO/913/90 from Junta Nacional de Investigação Científica e Tecnológica (JNICT) and by Instituto Nacional de Investigação Científica (INIC). J.H.L. acknowledges a BD grant from JNICT.

\section{References}

Alves, M. J., Martins, L. O. \& SÁ-Correia, I. (1991). Temperature profiles and alginate synthesis in mucoid and non-mucoid variants of Pseudomonas aeruginosa. Letters in Applied Microbiology 12, 244-248.

Bayer, A. S., Eftekar, F., Tu, J., Nast, C. C. \& Speert, D. P. (1990). Oxygen up-regulation of mucoid exopolysaccharide (alginate) production in Pseudomonas aeruginosa. Infection and Immunity 58, $1344-1349$

Deretic, V., Gill, J. F. \& Chakrabarty, A. M. (1987). Gene algD coding for GDP-mannose dehydrogenase is transcriptionally activated in mucoid Pseudomonas aeruginosa. Journal of Bacteriology 169, 351-358.

Deretic, V., Govan, J. R. W., Koniecsni, W. M. \& Martin, D. W. (1990). Mucoid Pseudomonas aeruginosa in cystic fibrosis: mutations in the muc loci affect transcription of the $\operatorname{alg} R$ and $\operatorname{alg} D$ genes in response to environmental stimuli. Molecular Microbiology 4, 189-196.

Deretic, V., Mohr, C. D. \& Martin, D. W. (1991). Mucoid Pseudomonas aeruginosa in cystic fibrosis: signal transduction and histone-like elements in the regulation of bacterial virulence. Molecular Microbiology 5, 1577-1583.

DeVault, J. D., Berry, A., Misra, T. K., Darzins, A. \& ChakraBARTY, A. M. (1989). Environmental sensory signals and microbial pathogenesis: Pseudomonas aeruginosa in cystic fibrosis. Bio/Technology 7, 352-357.

DeVault, J. D., Hendrickson, W., Kato, J. \& Chakrabarty, A. M. (1991). Environmentally regulated $\operatorname{alg} D$ promoter is responsive to the cAMP receptor protein in Escherichia coli. Molecular Microbiology 5, 2503-2509.

Estell, D. A., Graycar, T. P. \& Wells, J. A. (1985). Engineering an enzyme by site-directed mutagenesis to be resistant to chemical oxidation. Journal of Biological Chemistry 260, 6518-6521.

KNUTSON, C. A. \& JEANES, A. (1968). A new modification of the 
carbazole analysis: application to heteropolysaccharides. Analytical Biochemistry 24, 470-481.

Leitão, J. H., Fialho, A. M. \& Sá-Correia, I. (1992). Effects of growth temperature on alginate synthesis and enzymes in Pseudomonas aeruginosa variants. Journal of General Microbiology, 138, 605-610.

Martins, L. O., Brito, L. C. \& SÁ-Correia, I., (1990). Roles of $\mathrm{Mn}^{2+}$, $\mathrm{Mg}^{2+}$ and $\mathrm{Ca}^{2+}$ on alginate biosynthesis by Pseudomonas aeruginosa. Enzyme and Microbial Technology 12, 794-799.

Nosoh, Y. \& SekiguchI, T. (1991). Protein Stability and Stabilization through Protein Engineering. New York: Ellis Horwood.

Sá-Correia, I., Darzins, A., Wang, S.-K., Berry, A. \& Chakra-
BARTY, A. M. (1987). Alginate biosynthetic enzymes in mucoid and nonmucoid Pseudomonas aeruginosa: overproduction of phosphomannose isomerase, phosphomannomutase and GDP-mannose pyrophosphorylase by overexpression of phosphomannose isomerase (pmi) gene. Journal of Bacteriology, 169, 3224-3231.

Shinabarger, D., Berry, A., May, T. B., Rothmel, R. Fialho, A. M. \& ChaKrabaRTY, A. M. (1991). Purification and characterization of phosphomannose isomerase-guanosine diphospho-D-mannose pyrophosphorylase. Journal of Biological Chemistry, 266, 2080-2088.

SuTHERLAND, I. W. (1990). Biotechnology of Microbial Exopolysaccharides. Cambridge: Cambridge University Press. 